on this signal in an effort to lower the kinescope background, all of the scintillations would decrease equally in brightness both within the area occupied by a star image and in the background surrounding it. Under these circumstances, none of the sky background could be subtracted without weakening the wanted images to the same degree. In other words, the contrast could not be enhanced at all if perfect resolution were preserved throughout the system. As a result, the information storage capacity of the system would need to accommodate all of the sky background in addition to the images superposed upon it.

If, on the other hand, the charge resulting from each photo-electron is spread over a sufficient area to blend considerably into neighbouring charges, the output signal of the image orthicon will consist of a continuous background with small fluctuations. Under these circumstances, most of the background can be subtracted before the final picture is recorded, say, by photographing the kinescope. This subtraction trick provides an opportunity for increasing the integration capacity of the system, because most of the sky background does not need to be stored after the signal is removed from the target.

One can therefore envisage a two-step process of image information integration. The image orthicon is defocused to a degree where its resolution is equal to the minimum detail to be preserved in the picture. The target is scanned at intervals of every few minutes so that appreciable charge integration is obtained, but the storage capacity of the target is not exceeded. The output signal is biased to a degree where most of the background is discarded and only the residual portion containing the statistical fluctuations is delivered to the screen of the kinescope. These background-clipped pictures which are flashed periodically on the kinescope can be integrated photographically in one long exposure until the storage capacity of the photographic plate is used up.

It is difficult to calculate theoretically the gain in information storage that is achieved by this two-step procedure, because various non-fundamental effects would undoubtedly be encountered in a system so complex as the one just outlined. However, two or three orders of magnitude in storage capacity might be obtainable by this technique. Almost no experimental work of this nature has been done, and there is little point in trying to estimate possible performance more accurately until tests can be made.

An effort has been made in this paper to point out some of the ways in which present television techniques and their logical extensions may be of value in the field of astronomy. It is evident that much experimental and developmental work is needed in this field. For example, a tube could be designed which would filter out the unwanted uniform background information and thus conserve valuable storage capacity. Such a tube would make the two-step process outlined above unnecessary, yet it would accomplish the same result. This tube could be designed and operated in such a way that in addition to giving background filtering it would also give continuous presentation of the stored information. The development of this and other tubes which might be of considerable astronomical value can come only as the result of adequate theoretical and experimental research.

\title{
3. THE DETECTION OF FAINT IMAGES AGAINST THE SKY BACKGROUND
}

\section{By W. A. BAum, Mount Wilson and Palomar Observatories}

The detection of fainter and fainter objects in the sky has been of fundamental importance to modern advances in observational astronomy and cosmology, and it has led to the building of bigger and bigger telescopes in an effort to collect more and more light. A point has now been reached where the cost of building telescopes appreciably bigger than those now in existence becomes prohibitive, but the perfection of better image receivers for the light already available stands a good chance of accomplishing the same thing at comparatively modest expense. 
The features which an image receiver must have in order to provide an improvement in the detection of faint images can be best appreciated if the factors which presently limit conventional photography are considered. It will also be informative to evaluate these factors in terms of a particular case and then later to extend our thinking to general formulae.

Suppose, for example, that we want to photograph a certain field of stars at the prime focus of the 200 in. telescope at Palomar. If an ordinary camera shutter is placed in front of an Eastman ro3a-O plate and a 'snapshot' of this field is taken (say, 0.0I sec.), stars around eleventh magnitude will produce tiny grey images which are barely detectable. If the exposure is doubled, stars about twice as faint as before will produce similar images. Doubling the exposure again will add still another factor of two in detectability, and so on, up to a certain limit. It would be very nice if we could say 'and so on, indefinitely'; one could then reach almost any degree of faintness desired simply by having the patience to expose the plate long enough. Unfortunately, a levelling off in image detectability is encountered after only a few minutes. After an exposure of $30 \mathrm{~min}$., a 200 in. photograph shows about as much as it is going to, and further exposure is more likely to result in a loss rather than a gain.

The cause of this behaviour is not at all mysterious, but it tends to be forgotten when the supposed capabilities of the 200 in. telescope come into discussion, because it is less often encountered with smaller telescopes. Three well-known effects are involved: one is 'seeing', another is the night-sky glow, and the third is a 'full exposure' effect. A fourth effect, namely, the decrease of photographic efficiency due to reciprocity failure for very long exposures, is of relatively small importance in the present case.

The diameter and excursion of a stellar image formed by the 200 in. telescope is determined almost solely by turbulence in the Earth's atmosphere, that is, by 'seeing'. At the prime focus, images of $0.25 \mathrm{~mm}$. spread are average, images of $0 . \mathrm{I} \mathrm{mm}$. spread occur about one clear night in four, and images appreciably smaller than that are relatively rare. An image diameter of $0.1 \mathrm{~mm}$., which corresponds there to about I sec. of arc, is therefore fairly typical of favourable seeing conditions; this is several times larger than the resolving power of Eastman ro3a-O plates and 40 times larger than the optical resolving power of the telescope. It will be convenient here to define an area $0 \cdot \mathrm{Imm}$. in diameter as a 'picture element'.

The night-sky glow is a relatively. uniform luminous background against which the blurred stellar images must be detected. On a typical moonless night, the surface brightness of the sky is about fourth magnitude per square degree. Or, translated into terms of light falling onto a photographic plate at the prime focus of the 200 in., the night-sky glow provides a background illumination which is roughly equivalent to having a uniform compact mosaic of 22nd-magnitude star images, each covering one picture element. Wherever the image of an actual 22nd-magnitude star is superposed upon this background, the picture element occupied by the star receives just twice as much light as an empty background element. Picture elements occupied by fainter stars exceed the sky background by lesser amounts, and their detection depends accordingly upon whether the photographic emulsion is capable of distinguishing the surface brightness within the image element from the surface brightness of vacant background elements surrounding it.

A threshold image of the sort just described will be detected if the number of blackened photographic grains within the picture element it occupies exceeds the average number of blackened grains in equal-sized background elements by appreciably more than the statistical fluctuation in grain counts among the background elements. For an ideal emulsion, the percentage fluctuation among background elements should be inversely proportional to the square root of the average number of blackened grains per background element. In other words, the detection of a threshold photographic image depends simply upon the signal-to-noise ratio, which is a familiar concept in the electronics field.

For an actual emulsion, however, there is a 'full exposure' effect. The overlapping of blackened grains sets a limit on the number of statistically effective grains (that is, on 
the number of black and white granulations) which can populate a background element. It is for this reason that an Eastman roza-O plate at the prime focus of the 200 in. telescope reaches a so-called full exposure in about 30 min., beyond which further exposure does not materially improve the statistics of threshold image detection.

After allowance is made for this overlapping effect, grain counts indicate that we should theoretically expect the thresholds of Eastman ro3a-O plates which are fully exposed at the prime focus of the 200 in. telescope in good seeing to lie somewhere between $m_{p g}=23.5$ and 24.0 magnitude. During the past two years it has been possible to obtain a reliable observational check on this threshold by measuring the magnitudes of very faint objects directly at the telescope with a photo-electric photon-counting photometer. It has even been possible to obtain photo-electric observations of objects

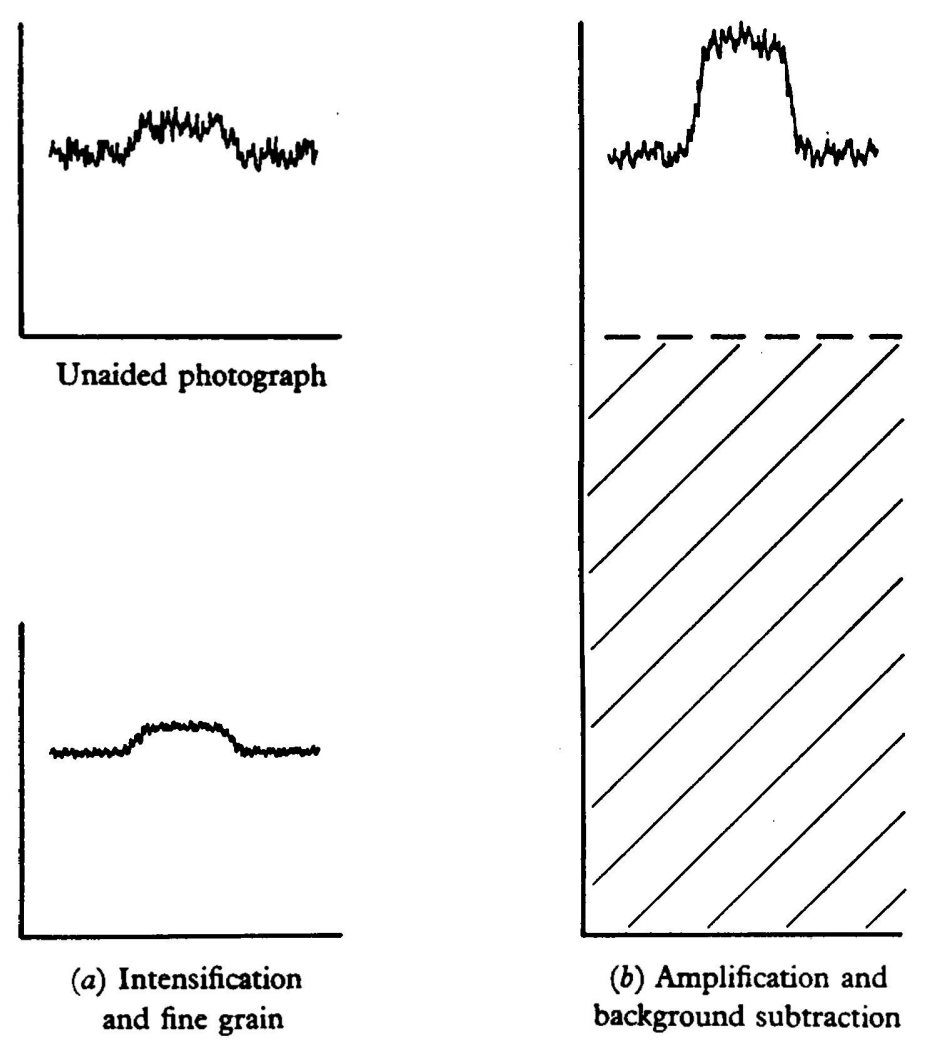

Fig. 4. Two general methods by which the signal-to-noise ratio can be increased so as to improve the discrimination of faint images against the sky background. These methods, both of which are aimed at avoiding the present limit set by statistical saturation, require photo-electric image tubes for their practical realization. Image converters (paper no. $\mathrm{r}$ ) represent method (a), while image-storage tubes (paper no. 2) represent method (b).

whose photographic images lie below the threshold; this can be done, for example, by making a photo-electric measurement of the blue magnitude of a red object whose position is determined from red photographs, but whose blue image cannot be photographically recognized. The faintest object which has thus far been detected on an Eastman ro3a-O plate was found photo-electrically to be $m_{p g}=23.8$ magnitude.

Since the percentage fluctuation in background grain counts is inversely proportional to the square root of the effective number of grains or granulations per background picture element, the only way to extend the present threshold is to produce more grains (or more photo-events of some sort) per element and at the same time avoid statistical saturation due to the overlapping of grains. As illustrated in Fig. 4, this can be accomplished either $(a)$ by making the grains smaller, or $(b)$ by subtracting a uniform excess 
portion of the background. In both cases, the basic aim is to permit the picture to be registered with a higher signal-to-noise ratio. For practical reasons, both methods call for electronic techniques, because the utilization of very fine-grain plates (method $a$ ) would entail prohibitively long photographic exposures if no intensification were employed, and because the background subtraction trick (method $b$ ) does not seem to be photographically feasible.

How, then, can we produce more grains per picture element? In unaided photography, one grain is blackened for every thousand eligible photons incident upon the emulsion during a full exposure of the type described above; the effective quantum efficiency is therefore only $0 \cdot 1 \%$. 'Eligible' here means within the wave-length range to which the emulsion is potentially sensitive. Electronic image tubes are very much better than that. For example, one electron is ejected for every ten eligible photons incident upon a photoemissive cathode; the quantum efficiency is therefore $10 \%$, which is Ioo times more efficient than the photographic emulsion. If, in turn, each of these photo-electrons is caused to produce one photographic grain, the combined process is roo times more efficient than unaided photography. Not only is this possible, but it has already been done; the problem is how to do it best for astronomical purposes and whether to utilize method $a$ or method $b$. Image-converter systems, which have been discussed in paper no. I by Prof. Lallemand, represent method $a$; while image-storage systems, which have been discussed in paper no. 2 by Dr Morton, represent method $b$.

Whatever the type of image-receiving system used, its threshold can be expressed in terms of various parameters. The fundamental quantities are the following:

$n=$ average number of eligible unscattered photons per second received per unit area at the surface of the Earth from a faint star.

$N=$ average number of eligible photons per second received per unit area at the surface of the Earth from unit solid angle of the sky background.

$t=$ effective integration time.

$q=$ average quantum efficiency in terms of independent registered events per eligible photon received during an exposure. For a photo-emissive cathode $q$ is not a function of $t$, but for a photographic emulsion it is.

$\beta=$ average number of instrument-background events per second per unit area (referred to the optical focus of the telescope) during an exposure; for example, thermionic emission from a photocathode, or the time-average of chemical fog in a photographic emulsion.

$k=\mathrm{a}$ coefficient of recognition, or a coefficient of certainty, which relates the actual threshold of recognition to the statistical uncertainty expressed as a standard deviation.

$\alpha=$ angular diameter of the star image (due mostly to seeing if the telescope is large).

$D=$ aperture of the telescope.

$f=$ focal length of the telescope.

$m=$ magnitude of the star within the wave-length band of the system.

$M=$ magnitude per unit solid angle of the sky background within the same wavelength band.

It will be convenient to neglect the factor $\pi / 4$, which partly cancels out of the main results anyway.

The total number of photo-events (such as blackened grains) due to the faint star will be $n D^{2} q t$ and the average number of background events within an equal area (i.e. within each picture element) will be $\left(N D^{2} q+\beta f^{2}\right) \alpha^{2} t$. Since these quantities will in general be numerically large, the threshold value of $n D^{2} q t$ should be

$$
\left(n D^{2} q t\right)_{0}=k\left[2\left(N D^{2} q+\beta f^{2}\right) \alpha^{2} t+n D^{2} q t\right]^{\frac{1}{2}} .
$$

This formula holds if all photo-events are truly independent and statistically effective, but the threshold will be less favourable if there is an appreciable degree of statistical saturation such as that introduced by the overlapping of photographic grains. An 
approximate way of taking this saturation effect into account is to regard $t$ as an effective exposure time which lags behind the actual exposure time on long exposures and which approaches an effective limit asymptotically.

The threshold contrast of surface brightness between the picture element occupied by the star image and its surroundings will simply be equation (I) divided by the number of background events per picture element, $\left(N D^{2} q+\beta f^{2}\right) \alpha^{2} t$. Since $n \ll N a^{2}$ near the threshold,

$$
\text { (Contrast })_{0} \approx\left[\frac{2 k^{2}}{\left(N D^{2} q+\beta f^{2}\right) \alpha^{2} t}\right]^{\frac{1}{2}},
$$

which can be simplified still further if the contribution of the instrument background $\beta f^{2}$ is also small compared with the contribution of the sky background $N D^{2} q$.

The magnitude of a star whose image is at the threshold will be given by

$$
\left.m_{0}=M-2.5 \log (n / N)_{0}=M-2.5 \log \left[\alpha^{2} \text { (Contrast }\right)_{0}(\mathrm{x}+R)\right],
$$

where $R$ is the ratio of instrument background to sky background and is likely to be small. The substitution of (2) into (3) yields

$$
m_{0} \approx \text { Constant }+0.5 M-2.5 \log \alpha-2.5 \log k+\mathrm{I} \cdot 25 \log \left(D^{2} q t\right)-\mathrm{I} \cdot 25 \log (\mathrm{I}+R),
$$

which is the final formula for the unsaturated threshold. This formula says, for example, that the threshold will be one magnitude fainter if the sky becomes two magnitudes less bright or if the image diameter due to seeing decreases by a factor of 2.5 I (i.e. by one magnitude). When expressed on a linear scale instead of a magnitude scale, the coefficient of one-half associated with the sky magnitude means that the threshold for image recognition depends upon the square-root of the surface brightness of the night sky, provided that the instrument background is small and provided that statistical saturation is not approached. This condition should apply not only to unsaturated image-tube observations but also to conventional photo-electric photometry, to visual observations with the human eye, and to ordinary lightly exposed photographs. The photo-electric case is in good agreement with photon-counting results obtained at Palomar, and the visual case provides an explanation for the square-root relationship already observed by Bowen, but the photographic case is presently of limited applicability because there is a rather restricted range of photographic exposures within which statistical saturation and instrument background are both small.

The formula for $m_{0}$ also says that an improvement in quantum efficiency $q$ has the same beneficial effect upon the threshold as increasing the area $D^{2}$ of the telescope aperture or increasing the effective integration time $t$. Therefore, if the full roo-fold advantage of photo-electric quantum efficiency could be ideally utilized in practice, one could attain the same threshold magnitude with an image tube at the 200 in. telescope as he could expect to reach by unaided photography (in the same exposure time) with a $2000 \mathrm{in}$. telescope! When we recall the historic accomplishments of the various large telescopes that exist today, and when we realize how much effort was expended in making each of them a working reality, we can appreciate the enormous potential importance of exploiting image tubes to the limit of technical ability. An actual practical gain amounting to only a small fraction of the theoretical goal would be worth a big effort. It would also accomplish much more than building a bigger telescope, because it would simultaneously benefit dozens of telescopes all over the world.

The ultimate threshold of a system is approached when the effective exposure $t$ approaches its limiting value due to the finite statistical capacity of the medium upon which the picture is registered. Let $E$ represent the maximum number of statistically effective photo-events which can populate a unit area at the primary focus. This means that the saturated value of $\left(N D^{2} q+\beta f^{2}\right) \alpha^{2} t$ is simply $\alpha^{2} f^{2} E$. Substituting this into equations (2) and (3), we obtain the statistically saturated threshold,

$$
m_{s} \approx M-2 \cdot 5 \log \alpha-2 \cdot 5 \log k+2 \cdot 5 \log f+\mathrm{I} \cdot 25 \log \frac{E}{2}-2 \cdot 5 \log (\mathrm{I}+R) .
$$


In this case, the threshold varies directly with sky brightness, but seeing has the same effect as before. The most remarkable feature of this saturated case is that the ultimate threshold does not depend upon the telescope aperture; it is a function of focal length only. It also does not depend upon the quantum efficiency of the receiver nor upon the time required for this statistical saturation to be reached.

Although image-tube observations have not yet been obtained with large telescopes, ordinary photographs provide a good quantitative test of formula (5). As an example, we can compute the theoretical threshold for an Eastman ro3 $a-0$ plate which is 'fully exposed' at the prime focus of the 200 in. telescope during good seeing on a moonless night. Take $M \approx-4.4$ magnitude per steradian, $\alpha \approx 5 \times 10^{-6}$ radians, $f=1855 \mathrm{~cm}$., and $E \approx 5 \times 10^{6}$ granulations per $\mathrm{cm} .{ }^{2}$. For a coefficient of certainty $k=3$, the predicted threshold $m_{s} \approx 23.8$ magnitude (blue), which is in excellent agreement with the photoelectrically checked value of the extreme photographic threshold already mentioned. A more conservative threshold, corresponding to $k=4$ and representing nearly 100\% certainty of recognition, is perhaps around 23.5 magnitude.

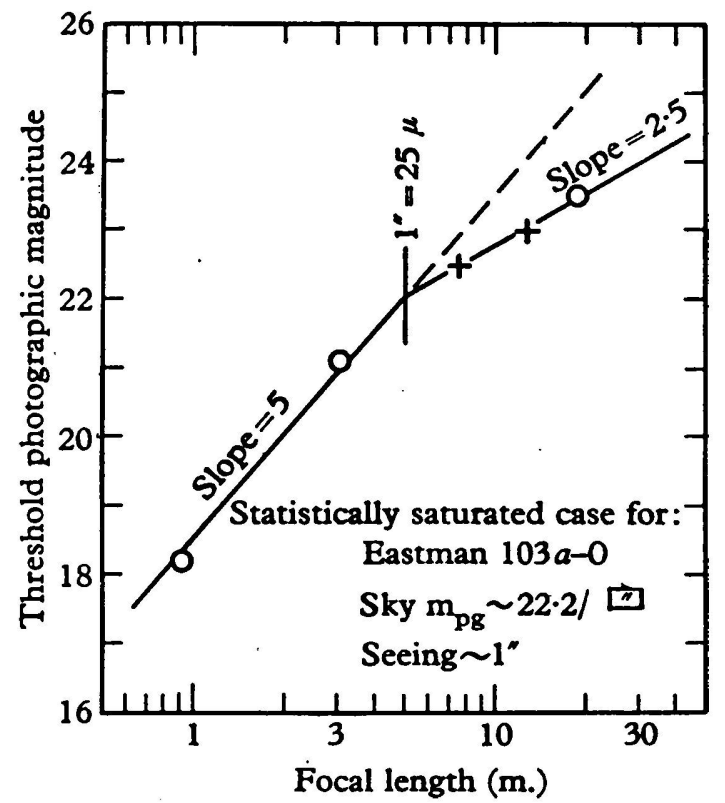

Fig. 5. Relationship between the limiting photographic magnitude and the focal length of a telescope. The points represent the observed limits of the five telescopes at Mount Wilson and Palomar, while the solid lines represent theoretical prediction. The data were all adjusted to a particular set of standard conditions.

Formulae (4) and (5) are derived on the assumption that seeing is the predominant factor in determining the size of a stellar image, and this is certainly the case for all large telescopes. For small telescopes, however, the effective angular image diameter $\alpha$ depends upon the focal length due to the finite resolving power of the emulsion (or equivalent); specifically, $\alpha$ varies as I / $f$. In formula (5) the $\alpha$ and $f$ terms then combine to give $+5 \log f$, which is the threshold dependence most commonly quoted.

Fig. 5 shows a rough comparison of saturated photographic thresholds for the principal telescopes at Mount Wilson and Palomar; they are plotted here against their focal lengths on a logarithmic scale. The three circles represent the $\mathrm{I} 8 \mathrm{in}$. Schmidt, the $48 \mathrm{in}$. Schmidt, and the 200 in. reflector at Palomar, while the two crosses represent the 60 in. and roo in. reflectors at Mount Wilson. The observed thresholds have all been chosen for (or adjusted to) a particular set of conditions so that all parameters except focal length are effectively identical; specifically, these conditions include a night-sky brightness of $22 \cdot 2$ blue magnitude per square second of arc, a seeing of I second of arc, a certainty of recognition 
close to $100 \%$, and the use of Eastman Io3 $a-0$ emilsion. Owing to the proximity of the city lights of Los Angeles and Pasadena, the two Mount Wilson telescopes (represented here by the crosses) never actually reach the extrapolated thresholds necessarily assigned to them in this diagram. Although it is difficult to specify saturated thresholds with any precision, the results here seem to be nicely consistent with theory. It is certainly evident, for example, that the line of slope 5, representing the conventional $5 \log f$ dependence for small telescopes, is grossly in error if extrapolated by the dashed line to long focal lengths. According to theory, the $2.5 \log f$ dependence should commence when the focal length becomes long enough for the minimum image diameter to be determined mainly by seeing rather than by the resolving power of the emulsion, and this is indeed just what we observe.

Not only are we already up against the statistically saturated case in conventional photography, but we shall also need to beware of it in predicting the performance of image-tube systems. Merely increasing the quantum efficiency $q$ of the receiver without increasing its storage capacity will gain nothing toward discriminating fainter images against the sky; an increase in $q$ is only a practical convenience which (through method $a$ or method $b$ ) makes the storing of more information feasible within reasonable exposure times. In principle, we should be able right now with no intensifying system to place a very fine-grain plate at the prime focus of the 200 in. telescope, expose it night after night to the same field, and eventually reach the same threshold of detection which an image-tube system would yield if its output were registered by method $a$ on the same plate. In practice, of course, reciprocity failure and the cost of telescope time would make such a procedure abortive.

Another limitation is encountered if a photographic emulsion is used for registering a photo-electric image by method $a$, as, for example, in Prof. Lallemand's system. If electrons strike the emulsion with an energy of several kilovolts, they will produce the order of one blackened grain per electron, but they will not penetrate more than a few per cent of the thickness of a typical emulsion. Consequently, the maximum photographic density which can be produced by low-energy electrons is very low; it is scarcely more than the sky background density which one would like to attain in order to approach the limit set by statistical saturation. This means, of course, that the contrast between images and sky, even for bright stars, would be very weak. If electrons are accelerated to higher energies so that they penetrate deeper into the emulsion, they each expose several grains instead of one and they thereby use up the additional storage capacity made available to them without actually storing more information. As a consequence, the emulsion approaches statistical saturation sooner and with a less favourable noise level. One must therefore compromise between the desire to produce a reasonable range of densities and the desire to utilize as much of the storage capacity of the emulsion as possible before being limited by statistical saturation.

To summarize: the image-discrimination threshold of a picture-receiving system is readily predictable on the basis of known parameters. The roles played by the quantum efficiency and the storage capacity of the system are of primary practical importance in seeking a fainter threshold. Photo-electric image tubes offer very bright hopes for the future of this problem, and there are two general methods $(a$ and $b$ ) by which the advantages of image tubes can be utilized.

\section{IMAGE CONVERTERS IN STELLAR SPECTROSCOPY}

\section{By W. A. HILTNER, Yerkes Observatory}

This paper is concerned primarily with the applications of image tubes to astronomical spectroscopy. The image tubes we now visualize may have their most extensive applications in that field. In this discussion we shall make the implicit assumption that a practical and efficient image converter or image-storage device is a reality and then 